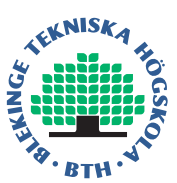

\title{
Effect of Primary Network on Performance of Spectrum Sharing System over Nakagami- $m$ Fading Channel
}

\author{
Fahim Khan \\ Pierre Celestin Mazarati \\ Nasir Khan
}

This thesis is presented as part of Degree of Masters of Science in Electrical Engineering with emphasis on Radio Communications

Blekinge Institute of Technology

September 2013

School of Engineering

Department of Electrical Engineering

Blekinge Institute of Technology, Sweden

Supervisor: Dr. Trung Q. Duong

Examiner: Prof. Dr.-Ing. Hans-Jürgen Zepernick 


\section{Contact Information:}

\section{Authors:}

Fahim Khan

email: fahimkhan.bth@gmail.com

Pierre Celestin Mazarati

email:mazopc@yahoo.fr

Nasir Khan

email: nasiriut@gmail.com

\section{Supervisor:}

Dr. Trung Q. Duong

Blekinge Institute of Technology, Sweden

email: quang.trung.duong@bth.se

\section{Examiner:}

Prof. Dr.-Ing. Hans-Jürgen Zepernick

Blekinge Institute of Technology, Sweden email: hans-jurgen.zepernick@bth.se 


\begin{abstract}
During the last few years, we have witnessed that radio spectrum is becoming a valuable and scarce resource due to the increasing demand of multimedia services. However, recent research has shown that most of the available radio spectrum is not used effectively and is wasted. So, to utilize the radio spectrum effectively, a new technology has been introduced known as "Cognitive Radio". In cognitive radio, a secondary user (SU) uses the vacant holes in licensed spectrum when it is not occupied by a primary user (PU) without causing interference to the PU transmission. Accessing vacant holes in the licensed user spectrum without causing interference to the PU is a complicated task. Therefore, alternative spectrum sharing techniques have gained popularity. Using these techniques, an SU can share the licensed spectrum with a PU at the same time without causing interference to the PU transmission.

As a result, a secondary user should have an optimal power allocation policy in order to get a high transmission rate while still keeping the interference caused to the primary user below a threshold value.

Under limited spectrum conditions, spectrum sharing relay networks have gained much popularity by providing reliability over direct transmission. In this thesis, we investigate an amplify-and-forward (AF) relay network performance in a spectrum sharing environment. Here, we consider the impact of the primary transmitter on the spectrum sharing system in the presence of a Nakagami- $m$ fading channel, where the fading parameter $m$ ( $m$ is an integer) can be used to deal with a variety of channel scenarios.
\end{abstract}





\section{Acknowledgements}

Our gratitude to Almighty God, the most merciful and the most gracious, who provided us strength and guidance to complete this thesis.

Our gratitude goes also to our honourable supervisor Dr. Quang Trung Duong for giving us an opportunity to work under his supervision. We really appreciate his patience and his friendly behaviour during our thesis work. Lastly, our special thanks go to our family members and friends for their support during our studies. 



\section{Contents}

1 Introduction $\quad 1$

1.1 Background of Cognitive Radio . . . . . . . . . . . . . . 1

1.1 .1 Background . . . . . . . . . . . . . . . . 1

1.1.2 Cognitive Radio . . . . . . . . . . . . . . . . . . . 1

1.1.3 Software Defined Radio . . . . . . . . . . . . . . . 2

1.1.4 Relation between Software Defined Radio and Cognitive Radio 3

1.1.5 Architecture of Cognitive Radio Network . . . . . . . . . . . 3

1.1.6 Spectrum Management . . . . . . . . . . . . . . 3

2 Cooperative Spectrum Sharing 5

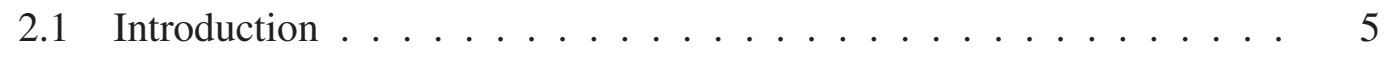

2.2 Cooperative Communications . . . . . . . . . . . . 5

2.3 Decode and Forward Method . . . . . . . . . . . . . . 6

2.4 Amplify and Forward Method . . . . . . . . . . . . . . 6

2.5 Cooperative Spectrum Sharing . . . . . . . . . . . . . . . 7

2.6 Classification of Spectrum Sharing . . . . . . . . . . . 8

3 System Model and Outage Probability Analysis 11

3.1 Introduction . . . . . . . . . . . . . . . . . 11

3.2 Nakagami- $m$ Fading Channel . . . . . . . . . . . . . . . . . . . 11

3.3 System Model . . . . . . . . . . . . . . . . . . . . . . . . . . 12

3.4 Numerical Results . . . . . . . . . . . . . . . . . . . . . . 17

4 Conclusion $\quad 21$ 



\section{Chapter 1}

\section{Introduction}

\subsection{Background of Cognitive Radio}

\subsubsection{Background}

Cognitive radio is a new and rising technology that enhances the already existing software-defined radio (SDR) [1]. Cognitive radio has the following two qualities [2]. First, it keeps an eye on the environment in which it exists. Second, it can change its physical layer characteristics based on current and previous experience with the environment. With these characteristics, the cognitive radio is capable of sensing the changing environment and is able to alter its physical layer behaviour such as channel selection, transmission power to fulfil the requirements of the users. The usage of radio spectrum is governed by the regulatory agencies such as the Federal Communication Commission (FCC) in the United States of America and the Office for Communications (OFCOM) in the United Kingdom. The regulatory agency allocates the radio spectrum to the licensed holders (primary users), on a long term basis. However, recent research has shown that most of the allocated spectrum is under-utilized. To avoid the inefficient use of radio spectrum, they come up with the idea of dynamic spectrum access (DSA) which enables the un-licensed users (secondary users) to use the under-utilized radio spectrum bands in the absence of licensed users (primary users). Cognitive radio is such a technology that can make use of the DSA technique in order to use the under-utilized radio spectrum [3].

\subsubsection{Cognitive Radio}

In the past few decades, we have witnessed a rapid growth in wireless technologies. As a result, the need for bandwidth and radio spectrum has increased tremendously. This limited spectrum poses some serious challenges to the continued growth in wireless communication. Recent research has shown that most of the radio spectrum remains unused. Cognitive radio is an emerging radio technology that can improve the efficiency of the radio spectrum utilization. Cognitive radio senses the vacant space in the radio spectrum by changing its operating parameters, uses the vacant spectrum in an opportunistic way and immediately vacate the spectrum bands once the primary user 
(PU) is detected. The cognitive radio efficiently uses radio spectrum by operating in both licensed and unlicensed bands and avoids the interference with the licensed or unlicensed users. The PU holds the license for a particular band and has the priority to use the channel. The secondary user (SU) can use the vacant bands as long as they do not affect the PU transmission. The following are the main functions of cognitive radio:

- Spectrum sensing

- Spectrum management

- Spectrum mobility

- Spectrum sharing

Cognitive radio is a revolutionary wireless technology having the capability to deal with scarcity of the radio spectrum. Cognitive radio is well aware of its surrounding radio environment. In order to maintain a good quality of service, the cognitive radio should learn from past experiences and its own current situation so that it can make a decision to fulfil the user requirements. In cognitive radio, spectrum sensing means to detect the availability of vacant bands in the radio spectrum. The SU uses the available radio spectrum bands and leaves the spectrum as soon as licensed users enter into the channel. The spectrum sensing in cognitive radio is cooperative. In cooperative sensing, those cognitive users who are near to the licensed users help the far away SU. After sensing the vacant bands in radio spectrum, the cognitive radio deals with the spectrum sharing. In general, spectrum sharing means, using the vacant bands in the radio spectrum in the absence of PUs. This process of sharing the system is cooperative spectrum sharing. Emerging technology such as cognitive radio has the capability to enable much more sharing of radio spectrum if we adopt some suitable radio spectrum policies. The idea of spectrum sharing reflects that a portion of radio spectrum assigned to a specific network or user can be used by another user on a temporary basis. This is accomplished in two ways: by reassigning allocated radio spectrum on temporary basis or by making possible the opportunistic use of available radio spectrum. The first method is used when the PU agrees to allow spectrum sharing. The second method is applicable when the users can find by themselves the vacant portion of the radio spectrum. The second method matches with the idea of cognitive radio. Finally after spectrum sharing, the cognitive radio reconfigures and transmits in the available radio spectrum band. During this process, the cognitive radio continuously changes its modulation scheme, carrier frequency and transmitting power.

\subsubsection{Software Defined Radio}

Wireless communication depends on the hardware and signals. In the old time, the wireless communication technology had very simple signalling, hardware and limited applications. To handle multiple wireless communication technologies (GSM, 
CDMA), the idea of SDR was put forward by researchers [4], so that the hardware can vary their operation according to the software. SDR has made the wireless communication devices capable of sensing, learning, monitoring and decision making. SDR has provided a base to implement the cognitive radio and has made it easy to understand its features [4].

\subsubsection{Relation between Software Defined Radio and Cognitive Ra- dio}

The cognitive radio is basically the same as SDR, it has the capability of cognition and is well aware of its surrounding environment. The SDR is basically based on digital signal processing with variable frequency components. The SDR is capable of operating over a number of different frequencies in multiple bandwidth. As a result of having such qualities, SDR can handle different standards like worldwide interoperability for microwave access (WiMAX), wideband code division multiple access (WCDMA), global system for mobile communications (GSM) etc, and different schemes like orthogonal frequency division multiplexing (OFDM), time division multiple access (TDMA) etc.

\subsubsection{Architecture of Cognitive Radio Network}

In wireless communication, a portion of radio spectrum is allocated to licensed users while the remaining part remains as unutilized. The PU network and SU network are the two major components of a cognitive radio network.

\section{Primary User}

Primary users are also known as licensed users and have the right to use some portion of radio frequency spectrum and their operation should not be affected by unlicensed users. Primary users allocate certain radio channels, the secondary users can make use of these channels assigned to PU under certain conditions [6]. According to IEEE 802.22 WRANs, television (TV) transmission towers are PUs, and radio devices that access TV channels are SUs.

\section{Secondary User}

SU holds no license to use the spectrum, and can only access the radio spectrum in an opportunistic way. The SUs should avoid interference to PUs transmission by leaving the channel immediately once they detect the PUs. SUs have the capabilities of spectrum sensing, spectrum decision and spectrum handoff.

\subsubsection{Spectrum Management}

In the presence of a primary network, a cognitive radio network faces some serious challenges. The cognitive radio network has to avoid interference with primary net- 
work and at the same time has to manage the best available radio spectrum band for transmission, to fullfil the requirements of quality of service (QoS). Spectrum management can be classified as follows:

\section{Spectrum Analysis}

In this method, every spectrum hole must be characterized by considering not only the time-varying radio frequency environment but also by the activity of the PU [7].

\section{Spectrum Decision}

After the analysis of the radio spectrum bands, the most suitable spectrum band should be selected considering the QoS requirements and the spectrum characteristics [7]. Based on user requirements on the data rate, bandwidth is chosen and a suitable radio band is determined on the base of a decision rule. 


\section{Chapter 2}

\section{Cooperative Spectrum Sharing}

\subsection{Introduction}

With the advancement of multimedia services, we see a large demand for bandwidth, higher data rates and radio spectrum. Therefore, communication engineers face many challenges meeting these demands. To overcome signal fading, shadowing effects and to meet the increasing demand of higher data rates and bandwidth, they have come up with the idea of cooperative communication where a direct link is accompanied by a relay node. The advantages of multiple-input multiple-output (MIMO) antenna systems have been widely acknowledged and have shown a tremendous performance improvement compared to single-antenna systems without using extra spectrum. A wireless system, especially handheld devices, is often not capable of handling more than one transmitting antenna due to the limitations of hardware, cost and size. To overcome this challenge and to benefit from performance enhancement by MIMO antenna systems, wireless communication engineers use cooperative communication techniques. The basic idea of cooperative communication is that single-antenna mobile devices form a virtual MIMO system by sharing their antennas among themselves in a multi-user scenario [5]. A single-antenna system cooperates with other single-antenna systems for transmitting information and this cooperation among them forms an array of virtual antennas and hence works as a transmitting channel for the source. Wireless networks face problems like shadowing and signal fading which degrades the signal significantly during its course of transmission. These problems can be easily overcome by transmitting independent copies of the signal from different location which generates diversity and we receive different copies of the transmitted signal at the receiver. This diversity can be generated by cooperative communications.

\subsection{Cooperative Communications}

In wireless cooperative communications, single-antenna devices enhance their quality of service through mutual cooperation. In such a communication system, each single wireless node assists other nodes in transmitting their data and thus acts as a part of a channel and hence each node transmits for multiple nodes. The concept of cooperative 


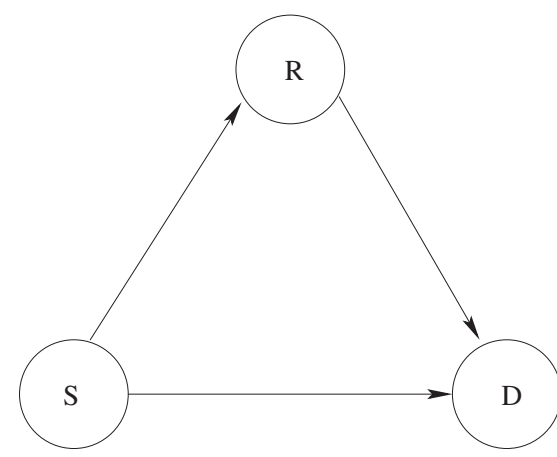

Figure 2.1: Basic relay newtwork.

communication can be traced back to three-terminal communication system: a source $\mathrm{S}$, receiver $\mathrm{D}$ and a relay $\mathrm{R}$ as shown in Fig. 2.1. However, in this network, the basic role of the relay is to assist the communication by transmitting data, whereas in cooperative communication each individual wireless node plays a multi-purpose role both as a relay and as a source of information. In cooperative communication, there are two different methods for signalling.

\subsection{Decode and Forward Method}

This method is closer to the concept of relay communication. In this protocol, the relay receives the signal from the source, decodes it and re-encodes it again before transmitting to the destination. The data sent by the source to the relay does not contain any information about the source-relay link and thus the processing of data makes it a hard decision at the relay. The decision taken at the relay influences the system performance. In case of decode-and-forward at the destination, this decision is not considered. This property of the decode-and-forward protocol affects the system performance. Many wrong decisions can be taken at the relay for an un-reliable source-relay link.

\subsection{Amplify and Forward Method}

In the amplify-and-forward (AF) protocol, the relay receives the signal transmitted by the source, amplifies it and retransmits it towards the destination. According to this protocol, it is a dual hop transmission. In the first hop, the relay receives the signal from the source and in the second hop the relay amplifies and forwards the signal towards the destination. In amplify-and-forward protocol, no re-generation of data is required which makes this protocol simple as compared to decode-and-forward protocol. 


\subsection{Cooperative Spectrum Sharing}

In spectrum sharing, the secondary user (SU) exists together with the primary user (PU) as long as the interference caused by the SU at the PU does not exceed the threshold. Software defined radios are wireless devices with a programmable processor [8]. Their cost is low, they can be upgraded easily and they can be deployed in real time. These wireless devices are capable of operating in multiple frequency bands under different transmission protocols and use different coding and modulation schemes. Cognitive radio improves the SDR and is well aware of its surrounding environment. It has the capability of making decisions without any intervention. This makes it capable of changing the transmission protocols and modulation schemes in order to communicate efficiently. Besides their benefits of less cost and flexibility, another advantage of SDR is spectral efficiency. A communication device like cognitive radio is capable of accessing the vacant frequency bands in the primary network for transferring its own data and this process can increase the effective usage of radio spectrum dramatically. This process of accessing the radio spectrum by cognitive radio is called sharing of spectrum [9]. Several protocols are proposed for spectrum sharing, where the secondary network senses and makes use of the vacant bands. Spectrum sharing consists of five steps which are the following:

- Spectrum Sensing:

If a cognitive radio wants to transmit its data, it should monitor the radio spectrum bands, analyze their information and then detect the spectrum holes. In cognitive radio, spectrum sensing is a vital technology. The spectrum sensing accuracy is a major factor to evaluate the performance of cognitive radio networks.

- Spectrum Allocation:

Once the spectrum hole is detected, the next step is to allocate the available radio spectrum band to the cognitive radio network.

- Spectrum Access:

Spectrum access helps to avoid collision and spectrum overlapping, when multiple nodes try to access spectrum.

- Transmitter Receiver Handshake:

In cognitive radio, once the spectrum is determined, the transmitter-receiver handshake is essential for effective transmission.

- Spectrum Mobility:

In cognitive radio, during transmission, spectrum mobility is very important between different secondary nodes. During transmission, if a specific portion is 


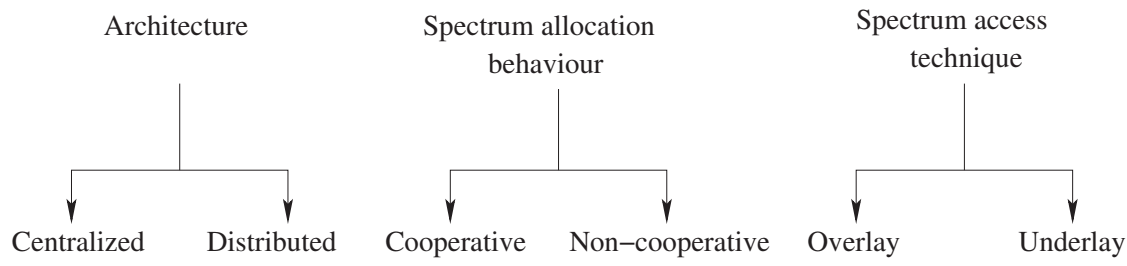

Figure 2.2: Classification of spectrum sharing.

required by the primary network, the secondary nodes should switch to a new radio spectrum band and should continue transmission.

\subsection{Classification of Spectrum Sharing}

Spectrum sharing can be divided into three main groups as shown in the Fig. 2.2.

\section{Centralized Spectrum Sharing}

The spectrum distribution and access procedures are supervised by a centralized entity and the measurements of spectrum distribution are forwarded to the central entity. This centralized server can rent out spectrum to users for limited time in limited geographical areas [10].

\section{Distributed Spectrum Sharing}

The spectrum distribution is based on locally available information that is performed by each user in a distributed way [10].

\section{Cooperative Spectrum Sharing}

The interference measurements of each node are utilized by cooperative solution and are shared among other nodes locally. The balance between centralized and distributed spectrum sharing is supplied by this local operation. [10]

\section{Non-cooperative Spectrum Sharing}

In non-cooperative spectrum sharing, a single node is taken into account as the interference information in other nodes is not considered, that is why it is called the selfish solution. The non-cooperative solution results in reduced spectrum utilization and does not need exchange of frequent messages between nodes [10]. 


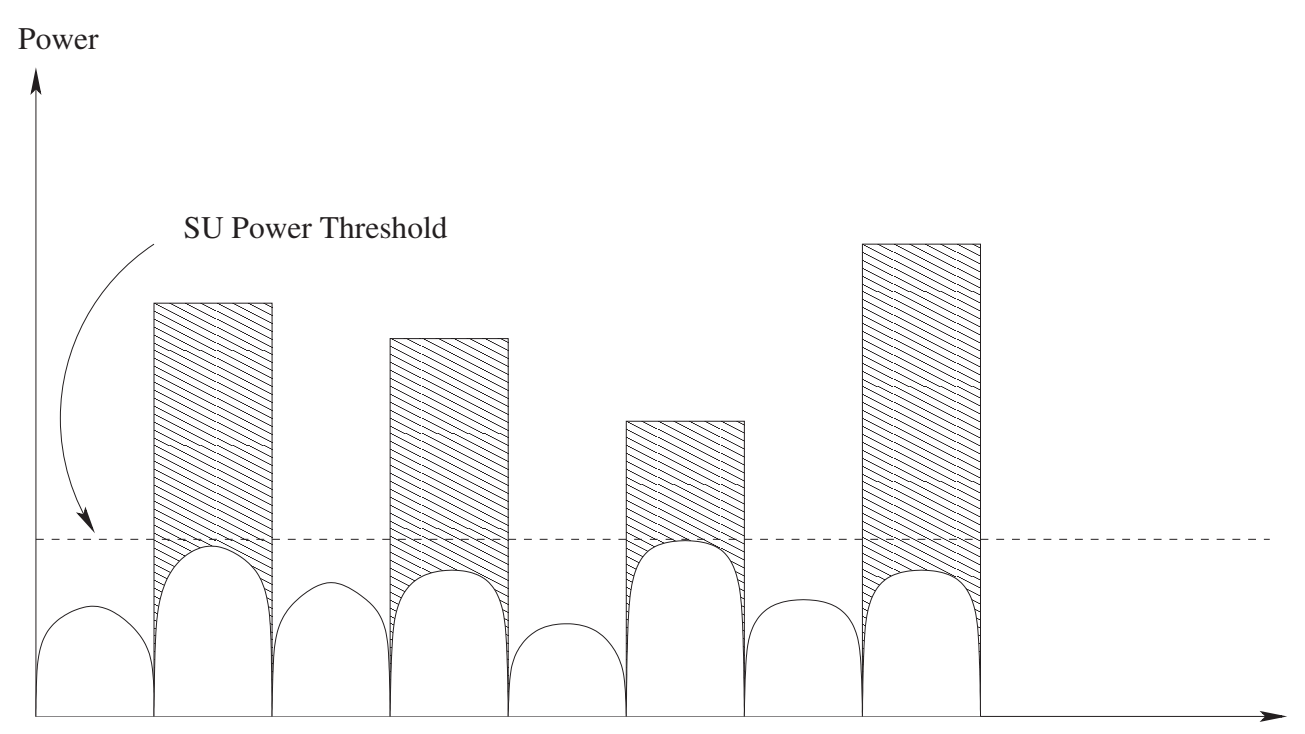

Frequency

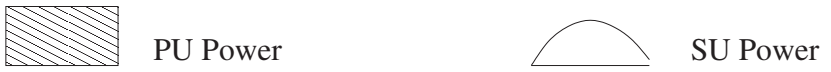

Figure 2.3: Underlay spectrum sharing.

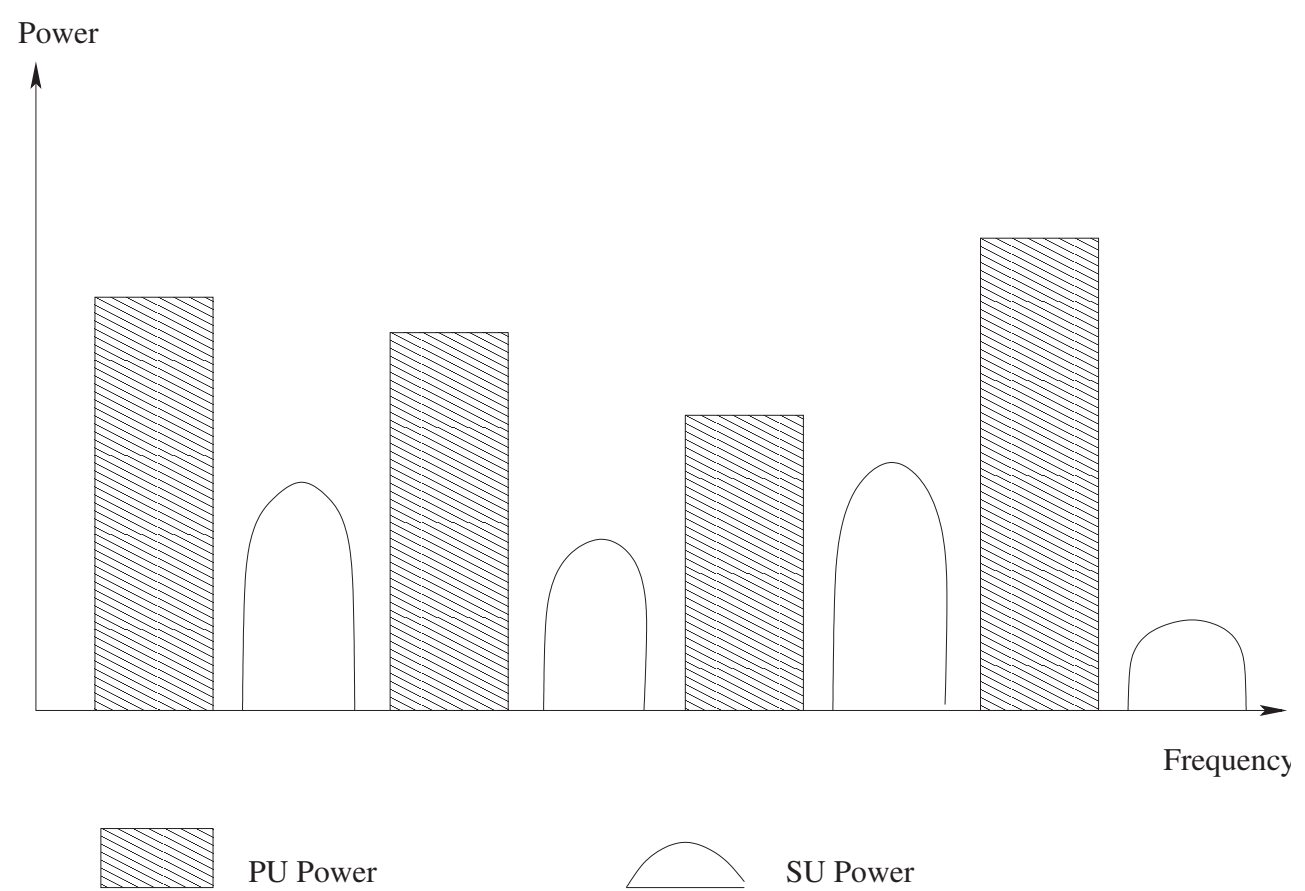

Figure 2.4: Interweave spectrum sharing.

\section{Overlay Spectrum Sharing}

In this system, the SU knows the codebooks and messages from the PU. These information is obtained as soon as the PU starts to transmit. With the information from 
the PU, the SU can use special techniques called dirty paper coding to entirely cancel the interference due to the PU. It can also use this information to set up its own communication rate. This method can be a solution to both SU and PU for performance improvement [11].

\section{Underlay Spectrum Sharing}

In this spectrum sharing technique, SU and PU can share the spectrum band simultaneously as long as the interference from the secondary network to the primary network transmission remains below a fixed threshold value [12]. To keep the interference below a certain threshold is a challenging job. In underlay spectrum sharing, the secondary network should meet the threshold condition even if the primary network is idle [13]. Applying the interference restrictions when the primary network is inactive reduces the transmission power of SU which affects its efficiency. Fig. 2.3 shows the underlay spectrum sharing.

\section{Interweave Spectrum Sharing}

In this method of spectrum sharing, the secondary network senses the vacant frequency bands in primary network intelligently and opportunistically starts to transmit its own data over the vacant band without causing any hamful interference to the PU. Once the PU is detected, the transmission of the SU is stopped immediately and the band should be left free for the PU. To use the available radio spectrum bands opportunistically, the current research is more interested in developing algorithms for secondary network [14]. Fig. 2.4 illustrates the interweave spectrum sharing. 


\section{Chapter 3}

\section{System Model and Outage Probability Analysis}

\subsection{Introduction}

In wireless communication, the transmitted signals suffer from delay, attenuation and phase shift due the presence of different reflectors in the environment in which transmitter and receiver operate. These reflectors give rise to multiple paths that a transmitted signal will go through, thus the receiver receives different replicas through all these paths. This is a main reason for loss in signal quality in wireless communication. To tackle this problem, the concept of antenna diversity is introduced. In antenna diversity techniques, multiple antennas take place of a single antenna, where a single path exists between each pair of transmitting and receiving antenna [15]. Antenna diversity plays a significant role in multipath situation. In antenna diversity, each pair of transmitting and receiving antenna experiences a unique fading environment. Thus, it is possible if one antenna suffers from more interference than the other, then that antenna with less interference will have a good quality of signal. This diversity scheme can be either spatial, temporal, frequency or antenna resources [16]. In spatial diversity, the signal is transmitted through various different paths. In case of wired transmission, the signals are transmitted through different wires while in the case of wireless transmission it is achieved by antenna diversity techniques. The signals are transmitted with different time slots in temporal diversity. In frequency diversity, the signals are transmitted using different frequency channels.

\subsection{Nakagami- $m$ Fading Channel}

There are different statistical models for studying different fading environments. For example, Rician and Rayleigh fading channels describe the fading environments in small geographical locations while Log-normal fading channel describes the fading environment for large geographical locations. The Nakagami- $m$ distribution is a probability distribution related to the gamma distribution. Nakagami- $m$ distribution is given by 


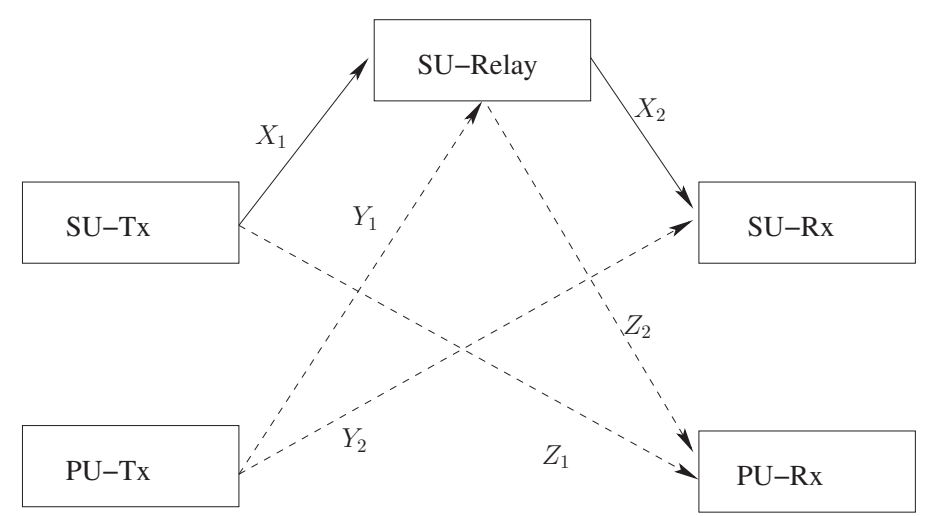

Figure 3.1: System model for spectrum sharing network considering interference from PU-Tx.

$$
p_{\gamma}(\gamma, m, \bar{\gamma})=\left(\frac{m}{\bar{\gamma}}\right) m \frac{\gamma^{m-1}}{\Gamma(m)} \exp \left(-\frac{m \gamma}{\bar{\gamma}}\right),
$$

where $m$ is the fading severity parameter and $\bar{\gamma}$ is the SNR. $m$ varies from 0.5 to $\infty$, $m=0.5$ gives one-sided Gaussian distribution while $m=1$ gives Rayleigh distribution.

\subsection{System Model}

In the last few years, the interest of the reseach community has increased tremendously in spectrum sharing relay networks due to its higher impact on direct transmission $[17,21]$. The results in $[17,21]$ have shown that using AF/DF relaying significantly improves the system performance in limited transmission power conditions. It has been shown in [19] that the effect from PU transmitter (PU-Tx) significantly affects the performance of the SU. In [21], a closed-form expression has been derived for outage probability in the case of Rayleigh fading channel.

In this thesis, we will consider Nakagami- $m$ fading channels. Let us consider a dualhop transmission system that is composed of an SU transmitter (SU-Tx), SU receiver (SU-Rx) and SU-relay in existence side by side with a primary network as shown in Fig. 3.1.

Let $S(t)$ be the signal transmitted by the SU-Tx to the SU-relay with the average power $P_{S}$ over the channel having a gain of $G$. The relay receives the signal $S_{r}$ from SU-Tx distorted by the PU transmission and is given by [21]:

$$
S_{r}=\sqrt{P_{s}} X_{1} s+\sqrt{P_{I}} Y_{1} x_{1}+n_{r}
$$


where $X_{1}$ is the channel coefficient for the link from SU-Tx to SU-relay, $P_{I}$ is PUTx average transmit power, $x_{1}$ is PU-Tx transmitted signal in the first hop and $n_{r}$ is additive white Gaussian noise (AWGN) at the SU-relay. After amplification with an amplifying gain $G$, the SU-relay forwards this received signal $S_{r}$ towards the destination with average power $P_{r}$. In the first and second hop, the transmission is done under power constraints $P_{s}$ and $P_{r}$ presented below:

$$
\begin{aligned}
& P_{s}=\frac{I_{p}}{\left|Z_{1}\right|^{2}}, \\
& P_{r}=\frac{I_{p}}{\left|Z_{2}\right|^{2}},
\end{aligned}
$$

where $I_{p}$ is the maximum torelable interference power. $Z_{1}$ and $Z_{2}$ are the channel coefficients of the interference links SU-Tx to PU-Rx and SU-relay to PU-Rx. The SU-Rx receives this signal and is given by [18]:

$$
S_{D}=\sqrt{P_{s}} G X_{1} s+G X_{2} n_{r}+G X_{2} \sqrt{P_{I}} Y_{1} x_{1}+n_{D}+\sqrt{P_{I}} Y_{2} x_{2},
$$

where $X_{2}$ and $Y_{2}$ are the channel coefficients for the link SU-relay to SU-Rx and PUTx to SU-Rx, $x_{2}$ is the transmitted signal of the link PU-Tx to SU-Rx and $n_{D}$ is the AWGN at the SU-Rx. By considering non-identical Nakagami-m fading in which all fading channel coefficients $\left|X_{1}\right|^{2},\left|X_{2}\right|^{2},\left|Y_{1}\right|^{2},\left|Y_{2}\right|^{2},\left|Z_{1}\right|^{2}$, and $\left|Z_{2}\right|^{2}$ are gamma distributed with fading severity parameters $m_{X_{1}}, m_{X_{2}}, m_{Y_{1}}, m_{Y_{2}}, m_{Z_{1}}$, and $m_{Z_{2}}$ and channel power of fading $\Omega_{X_{1}}, \Omega_{X_{2}}, \Omega_{Y_{1}}, \Omega_{Y_{2}}, \Omega_{Z_{1}}$ and $\Omega_{Z_{2}}$, respectively. The SNR at the destination (SU-Rx) can be written as

$$
\gamma_{D}=\frac{\frac{X_{1}}{Y_{1} Z_{1}} \frac{\bar{\gamma}}{\gamma_{I}} \frac{X_{2}}{Y_{2} Z_{2}} \frac{\bar{\gamma}}{X_{I}}}{\frac{\bar{\gamma}}{Y_{1} Z_{1}} \frac{X_{I}}{\gamma_{I}}+\frac{X_{2}}{Y_{2} Z_{2}} \frac{\bar{\gamma}}{\gamma}}=\frac{\gamma_{1} \gamma_{2}}{\gamma_{1}+\gamma_{2}}
$$

with

$$
\begin{aligned}
\gamma_{1} & =a \frac{X_{1}}{Y_{1} Z_{1}}, \\
\gamma_{2} & =a \frac{X_{2}}{Y_{2} Z_{2}},
\end{aligned}
$$

where $\frac{\bar{\gamma}}{\gamma_{I}}=a, \bar{\gamma}=\frac{I_{p}}{N_{0}}, \overline{\gamma_{I}}=\frac{P_{I}}{N_{0}}$ and $N_{0}$ representing the noise variance. According to [21], the upper bound for $\gamma_{D}$ given in (3.6) can be written as $\gamma_{D} \leq \gamma_{D \text { up }}=\min \left(\gamma_{1}, \gamma_{2}\right)$.

In order to obtain the outage probability, first we need to obtain the cumulative density function (CDF) of $U=\frac{X}{Y Z}$, where $X, Y$ and $Z$ are gamma distributed random variables with fading severity parameters $m_{X_{1}}, m_{X_{2}}, m_{Y_{1}}, m_{Y_{2}}, m_{Z_{1}}, m_{Z_{2}}$. The CDF can be derived from

$$
F_{\gamma_{1}}(\gamma)=\int_{0}^{\infty} \int_{0}^{\infty} F_{X_{1}}\left(\frac{\gamma}{a} y z\right) f_{y_{1}}(y) f_{z_{1}}(z) d y d z
$$


where the CDF and probability density function (PDF) of $X$, for $X \in\left\{X_{1}, X_{2}, Y_{1}\right.$, $\left.Y_{2}, Z_{1}, Z_{2}\right\}$, can be written as

$$
\begin{gathered}
f_{X}(x)=\frac{(\alpha)^{m}}{\Gamma(m)} x^{m-1} \exp (-\alpha x), \\
F_{X}=\frac{1}{\Gamma(m)} \gamma(m, \alpha x)=1-\frac{\Gamma(m, \alpha x)}{\Gamma(m)},
\end{gathered}
$$

where $\gamma(\cdot), \Gamma(\cdot, \cdot)$ represent the gamma function [19, Eq. (8.310.1)] and the upper incomplete gamma function [19, Eq. (8.350.2)] respectively, $\gamma(\cdot, \cdot)$ denotes the lower incomplete gamma function [19, Eq. (8.350.1)], where $\alpha \in\left\{\alpha_{X_{1}}=\frac{m_{x_{1}}}{\Omega_{x_{1}}}, \alpha_{x_{2}}=\right.$ $\left.\frac{m_{x_{2}}}{\Omega_{x_{2}}}, \alpha_{y_{1}}=\frac{m_{y_{1}}}{\Omega_{y_{1}}}, \alpha_{y_{2}}=\frac{m_{y_{2}}}{\Omega_{y_{2}}}, \alpha_{z_{1}}=\frac{m_{z_{1}}}{\Omega_{z_{1}}}, \alpha_{z_{2}}=\frac{m_{z_{2}}}{\Omega_{z_{2}}}\right\}$ and $m \in\left\{m_{x_{1}}, m_{x_{2}}, m_{y_{1}}\right.$, $\left.m_{y_{2}}, m_{z_{1}}, m_{z_{2}}\right\}$.

Equation (3.12) becomes

$$
F_{\gamma_{1}}(\gamma)=\int_{0}^{\infty} \int_{0}^{\infty} \frac{\Gamma\left(m_{x_{1}} \frac{\alpha_{X_{1}} \gamma y z}{a}\right)}{\Gamma\left(m_{x_{1}}\right)} f_{y_{1}}(y) f_{z_{1}}(z) d y d z
$$

where

$$
\frac{\Gamma\left(m_{x_{1}} \frac{\alpha_{X_{1}} \gamma y z}{a}\right)}{\Gamma\left(m_{x_{1}}\right)}=\mathrm{e}^{\frac{-\alpha_{X_{1}} \gamma y z}{a}} \sum_{i=0}^{m_{x_{1}-1}}\left(\frac{\alpha_{X_{1}} \gamma y z}{a}\right)^{i} \frac{1}{i !},
$$

where $\Gamma(\cdot, \cdot)$ is an incomplete gamma function [19,Eq.(8.352.2)]. The PDF of both $Y$ and $Z$, can be written as follows:

$$
\begin{aligned}
& f_{Y_{1}}(y)=\frac{\left(\alpha_{Y_{1}}\right)^{m_{y_{1}}}}{\Gamma\left(m_{y_{1}}\right)} y^{m_{y_{1}}-1} \exp \left(-\alpha_{Y_{1}} y\right), \\
& f_{Z_{1}}(z)=\frac{\left(\alpha_{Z_{1}}\right)^{m_{z_{1}}}}{\Gamma\left(m_{z_{1}}\right)} z^{m_{z_{1}}-1} \exp \left(-\alpha_{Z_{1}} z\right) .
\end{aligned}
$$

Substitute (3.15), (3.16) and (3.17) in (3.14) gives

$$
\begin{aligned}
F_{\gamma_{1}}(\gamma) & =1-\sum_{i=0}^{m_{x_{1}-1}}\left(\frac{\alpha_{X_{1}} \gamma}{a}\right)^{i} \frac{1}{i !} \frac{\alpha_{Y_{1}}^{m_{y_{1}}}}{\Gamma\left(m_{y_{1}}\right)} \frac{\alpha_{Z_{1}}^{m_{z_{1}}}}{\Gamma\left(m_{z_{1}}\right)} \\
& \times \int_{0}^{\infty} \int_{0}^{\infty}(y z)^{i} y^{m_{y_{1}-1}} \exp \left(-\alpha_{Y_{1}} y\right) z^{m_{z_{1}-1}} \\
& \times \exp \left(-\alpha_{Z_{1}} z\right) \exp \left(\frac{\alpha_{X_{1}} \gamma y z}{a}\right) d y d z
\end{aligned}
$$

Let us introduce 


$$
\begin{aligned}
I & =\int_{0}^{\infty} \int_{0}^{\infty}(y z)^{i} y^{m_{y_{1}-1}} \exp \left(-\alpha_{Y_{1}} y\right) z^{m_{z_{1}-1}} \exp \left(-\alpha_{Z_{1}} z\right) \\
& \times \exp \left(\frac{\alpha_{X_{1}} \gamma y z}{a}\right) d y d z
\end{aligned}
$$

Then,

$$
F_{\gamma_{1}}(\gamma)=1-\sum_{i=0}^{m_{x_{1}-1}}\left(\frac{\alpha_{X_{1}} \gamma}{a}\right)^{i} \frac{1}{i !} \frac{\alpha_{Y_{1}}^{m_{y_{1}}}}{\Gamma\left(m_{y_{1}}\right)} \frac{\alpha_{Z_{1}}^{m_{z_{1}}}}{\Gamma\left(m_{z_{1}}\right)} I
$$

where

$$
I=\int_{0}^{\infty} z^{m_{z_{1}+i-1}} \exp \left(-\alpha_{Z_{1}} z\right) I_{1}(y) d z
$$

and

$$
\begin{aligned}
I_{1}(y) & =\int_{0}^{\infty} y^{m_{y_{1}}+i-1} \exp \left(-\frac{\alpha_{X_{1}} \gamma z}{a}+\alpha_{Y_{1}} y\right) d y \\
& =\left(\frac{\alpha_{X_{1}} \gamma z}{a}+\alpha_{Y_{1}}\right)^{-m_{y_{1}}+i} \Gamma\left(m_{y_{1}}+1\right) .
\end{aligned}
$$

Substituting (3.23) into (3.21), we get

$$
I=\Gamma\left(m_{y_{1}}+i\right) \int_{0}^{\infty}\left(\frac{\alpha_{X_{1}} \gamma z}{a}+\alpha_{Y_{1}}\right)^{-m_{y_{1}}+i} z^{m_{z_{1}}+i-1} \exp \left(-\alpha_{Z_{1}} z\right) d z
$$

The above equation can be easily solved by using the Hypergeometric function [19, Eq. (9.211.4)], which gives us the following answer:

$$
\begin{aligned}
I & =\Gamma\left(m_{y_{1}}+i\right)\left(\frac{\alpha_{X_{1}} \gamma}{a}\right)^{-\left(m_{z_{1}}+i\right)}\left(\alpha_{y_{1}}\right)^{m_{z_{1}}-m_{y_{1}}} \\
& \times U\left(m_{z_{1}}+i, 1+m_{z_{1}}-m_{y_{1}}, a \frac{\alpha_{y_{1}} \alpha_{Z_{1}}}{\alpha_{X_{1}} \gamma}\right),
\end{aligned}
$$

where $U\left(\epsilon_{1}, \beta_{1}, t_{1}\right)$ is the Hypergeometric function [19, Eq. (9.211.4)] with $\epsilon_{1}=$ $m_{z_{1}}+i, \beta_{1}=1+m_{z_{1}}-m_{y_{1}}, t_{1}=a \frac{\alpha_{y_{1}} \alpha_{Z_{1}}}{\alpha_{X_{1}} \gamma}$. We can substitute (3.25) into (3.20) and the CDF of $\gamma_{1}$ is then obtained as

$$
\begin{aligned}
F_{\gamma_{1}}(\gamma) & =1-\sum_{i=0}^{m_{x_{1}}-1}\left(\frac{\alpha_{X_{1}} \gamma}{a}\right)^{i} \frac{1}{i !} \frac{\left(\alpha_{Y_{1}}\right)^{m_{y_{1}}}}{\Gamma\left(m_{y_{1}}\right)} \frac{\left(\alpha_{Z_{1}}\right)^{m_{z_{1}}}}{\Gamma\left(m_{z_{1}}\right)}\left(\frac{\alpha_{X_{1}} \gamma}{a}\right)^{-\left(m_{z_{1}}+i\right)} \\
& \times \alpha_{Y_{1}}{ }^{m_{z_{1}}-m_{y_{1}}} \Gamma\left(m_{y_{1}}+i\right) U\left(m_{z_{1}}+i, 1+m_{z_{1}}-m_{y_{1}}, a \frac{\alpha_{y_{1}} \alpha_{Z_{1}}}{\alpha_{X_{1}} \gamma}\right)
\end{aligned}
$$


The CDF of $\gamma$ in the first and second hop will be $F_{\gamma_{1}}(\gamma)$ and $F_{\gamma_{2}}(\gamma)$, where

$$
\begin{aligned}
& F_{\gamma_{1}}(\gamma)=1-A_{1}, \\
& F_{\gamma_{2}}(\gamma)=1-A_{2},
\end{aligned}
$$

and the outage probability (OP) at the destination is

$$
P_{\text {out }}=F_{\gamma_{D}}\left(\gamma_{t h}\right)
$$

where $\gamma_{t h}$ is the outage threshold. Then, $F_{\gamma_{D}}\left(\gamma_{t h}\right)$ can be calculated as

$$
\begin{gathered}
F_{\gamma_{D}}\left(\gamma_{t h}\right)=1-\left[1-F_{\gamma_{1}}\left(\gamma_{t h}\right)\right]\left[1-F_{\gamma_{2}}\left(\gamma_{t h}\right)\right], \\
F_{\gamma_{D}}\left(\gamma_{t h}\right)=1-A_{1} A_{2},
\end{gathered}
$$

where

$$
\begin{aligned}
& A_{1}=\sum_{i=0}^{m_{x_{1}}-1} \frac{\Gamma\left(m_{y_{1}}+i\right)}{\Gamma\left(m_{y_{1}}\right) \Gamma\left(m_{z_{1}}\right) i !} \Gamma\left(m_{z_{1}}+i\right) a \frac{\alpha_{Y_{1}} \alpha_{Z_{1}}}{\alpha_{X_{1}} \gamma} U\left(\epsilon_{1}, \beta_{1}, t_{1}\right), \\
& A_{2}=\sum_{i=0}^{m_{x_{2}}-1} \frac{\Gamma\left(m_{y_{2}}+i\right)}{\Gamma\left(m_{y_{2}}\right) \Gamma\left(m_{z_{2}}\right) i !} \Gamma\left(m_{z_{2}}+i\right) a \frac{\alpha_{Y_{2}} \alpha_{Z_{2}}}{\alpha_{X_{2}} \gamma} U\left(\epsilon_{2}, \beta_{2}, t_{2}\right),
\end{aligned}
$$

and $U\left(\epsilon_{1}, \beta_{1}, t_{1}\right)$ and $U\left(\epsilon_{2}, \beta_{2}, t_{2}\right)$ are the Hypergeometric functions for $F_{\gamma_{1}}\left(\gamma_{t h}\right)$ and $F_{\gamma_{2}}\left(\gamma_{t h}\right)$. Here, $\epsilon_{2}=m_{z_{2}}+i, \beta_{2}=1+m_{z_{2}}-m_{y_{2}}, t=a \frac{\alpha_{y_{2}} \alpha_{Z_{2}}}{\alpha_{X_{2}} \gamma}$

Therefore, an analytical expression of the OP for Nakagami- $m$ fading channels has been derived as shown by the above equations and derivation was based on the system model composed by SU transmitter and receiver, PU transmitter and receiver and AF relay. The above derivation was evaluated with the help of MATHEMATICA software. 


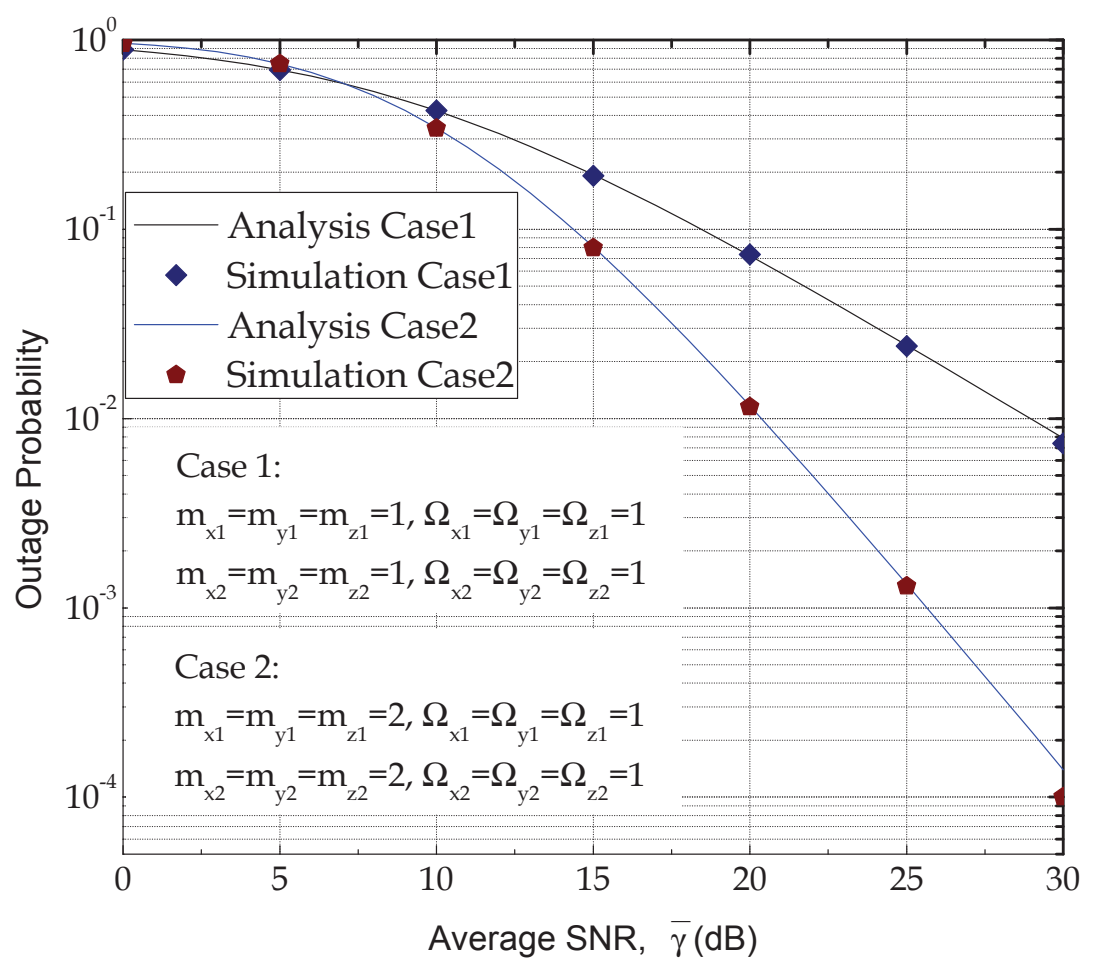

Figure 3.2: OP versus average SNR for Case1: $m_{X_{1}}=m_{X_{2}}=m_{Y_{1}}=m_{Y_{2}}=m_{Z_{1}}=m_{Z_{2}}=$ $1, \Omega_{X_{1}}=\Omega_{Y_{1}}=\Omega_{Z_{1}}=\Omega_{X_{2}}=\Omega_{Y_{2}}=\Omega_{Z_{2}}=1$, and Case2: $m_{X_{1}}=$ $m_{X_{2}}=m_{Y_{1}}=m_{Y_{2}}=m_{Z_{1}}=m_{Z_{2}}=2, \Omega_{X_{1}}=\Omega_{Y_{1}}=\Omega_{Z_{1}}=\Omega_{X_{2}}=\Omega_{Y_{2}}=\Omega_{Z_{2}}=1$

\subsection{Numerical Results}

In this section, numerical results presented in order to analyze the network's OP performance by showing the effects of interference from primary network on spectrum sharing system over Nakagami- $m$ fading channel. In this simulation, the outage threshold was set to $3 d B$ for all scenarios and the values of fading severity parameter and channel power are set depending on different scenarios which have been studied in this thesis. A match of results between analysis and simulation is observed. Figs. 3.2, 3.3 and 3.4 show the outage probability versus average SNR in $d B$. The PU transmitter and receiver parameters which are related to the interference links, are varied depending on the cases. All these figures represent the OP for spectrum sharing AF relay over Nakagami- $m$ fading channels and the primary network's performance effects on the secondary network has been studied.

Fig. 3.2 shows the outage probability versus the average SNR for the effect of primary network on performance of spectrum sharing system over Nakagami- $m$ fading channel when fading severity parameters and channel powers for both first and second hop in secondary and primary network are all equal to $1\left(m_{X_{1}}=m_{X_{2}}=m_{Y_{1}}=m_{Y_{2}}=\right.$ $m_{Z_{1}}=m_{Z_{2}}=1$ and $\Omega_{X_{1}}=\Omega_{X_{2}}=1, \Omega_{Y_{1}}=\Omega_{Z_{1}}=\Omega_{Y_{2}}=\Omega_{Z_{2}}=1$ ), in Case 1. In Case 2 , the fading severity parameters for secondary and primary network in both hops 


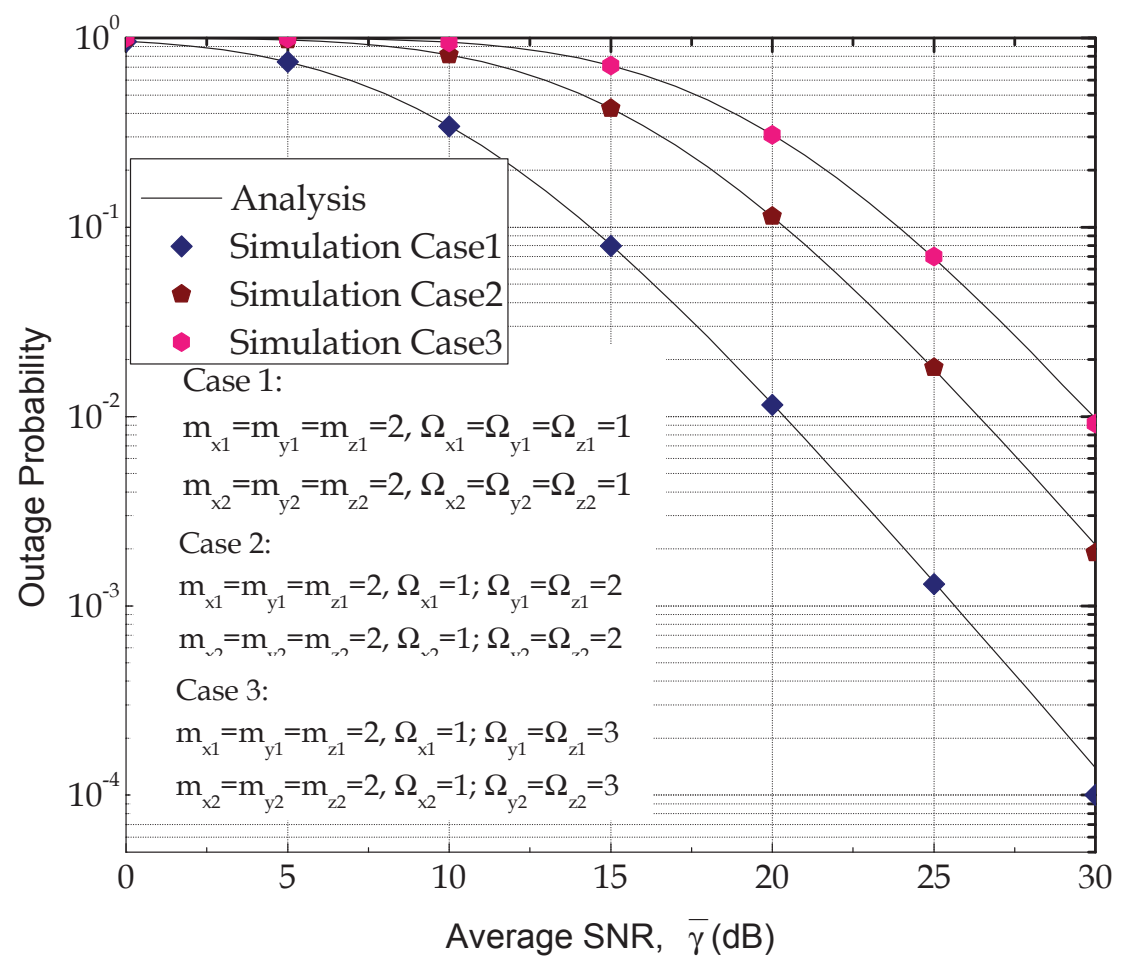

Figure 3.3: OP versus average SNR when Case1: $m_{X_{1}}=m_{X_{2}}=m_{Y_{1}}=m_{Y_{2}}=m_{Z_{1}}=m_{Z_{2}}=2$, $\Omega_{X_{1}}=\Omega_{Y_{1}}=\Omega_{Z_{1}}=\Omega_{X_{2}}=\Omega_{Y_{2}}=\Omega_{Z_{2}}=1$; Case2: $m_{X_{1}}=m_{X_{2}}=m_{Y_{1}}=m_{Y_{2}}=m_{Z_{1}}=m_{Z_{2}}=$ $2, \Omega_{X_{1}}=\Omega_{X_{2}}=1, \Omega_{Y_{1}}=\Omega_{Z_{1}}=\Omega_{Y_{2}}=\Omega_{Z_{2}}=2$ and Case3: $m_{X_{1}}=$ $m_{X_{2}}=m_{Y_{1}}=m_{Y_{2}}=m_{Z_{1}}=m_{Z_{2}}=2, \Omega_{X_{1}}=\Omega_{X_{2}}=1, \Omega_{Y_{1}}=\Omega_{Z_{1}}=\Omega_{Y_{2}}=\Omega_{Z_{2}}=3$

were increased to 2 and the channel powers were kept constant. By comparing the two cases, the curve of Case 2 has better performance of outage probability than the curve of Case 1 and the outage probability will vary depending to the point in consideration on the curve. Case 2 has high value of outage probability. With $m=1$, the Nakagami fading in this case will have Rayleigh fading behaviour while when we increase $m$ to 2 the fading at the SU-Tx will be less severe and the transmission will less degraded.

In Fig. 3.3, fading severity parameters were increased to 2 and channel powers were fixed to $1\left(m_{X_{1}}=m_{X_{2}}=m_{Y_{1}}=m_{Y_{2}}=m_{Z_{1}}=m_{Z_{2}}=2\right.$ and $\Omega_{X_{1}}=\Omega_{X_{2}}=1, \Omega_{Y_{1}}=$ $\Omega_{Z_{1}}=\Omega_{Y_{2}}=\Omega_{Z_{2}}=1$ ), in Case 1 . In Case 2 , only channel powers $\Omega_{Y_{1}}, \Omega_{Z_{1}}, \Omega_{Y_{2}}, \Omega_{Z_{2}}$ were increased to 2 while $\Omega_{X_{1}}, \Omega_{X_{2}}$ were fixed to 1 . In Case $3, \Omega_{X_{1}}, \Omega_{X_{2}}$ remained constant and channel powers linked to interference $\Omega_{Y_{1}}, \Omega_{Z_{1}}, \Omega_{Y_{2}}, \Omega_{Z_{2}}$ were increased to 3. The result shows that Case 1 performs better than Case 2 and Case 3. For outage probability of $10^{-2}$, we can see that the Case 1 has average SNR of $20.1 \mathrm{~dB}$ while Case 2 has average SNR of $25.3 \mathrm{~dB}$ and Case 3 with an average SNR of $30 \mathrm{~dB}$. In Case 2 and Case 3, we increased the value of channel power of the interference links $Y$ and $\mathrm{Z}$ to 2 and then 3. The performance with these channel powers were not as good as in Case 1 where the channel powers linked to interference is 1 . By increasing the chan- 


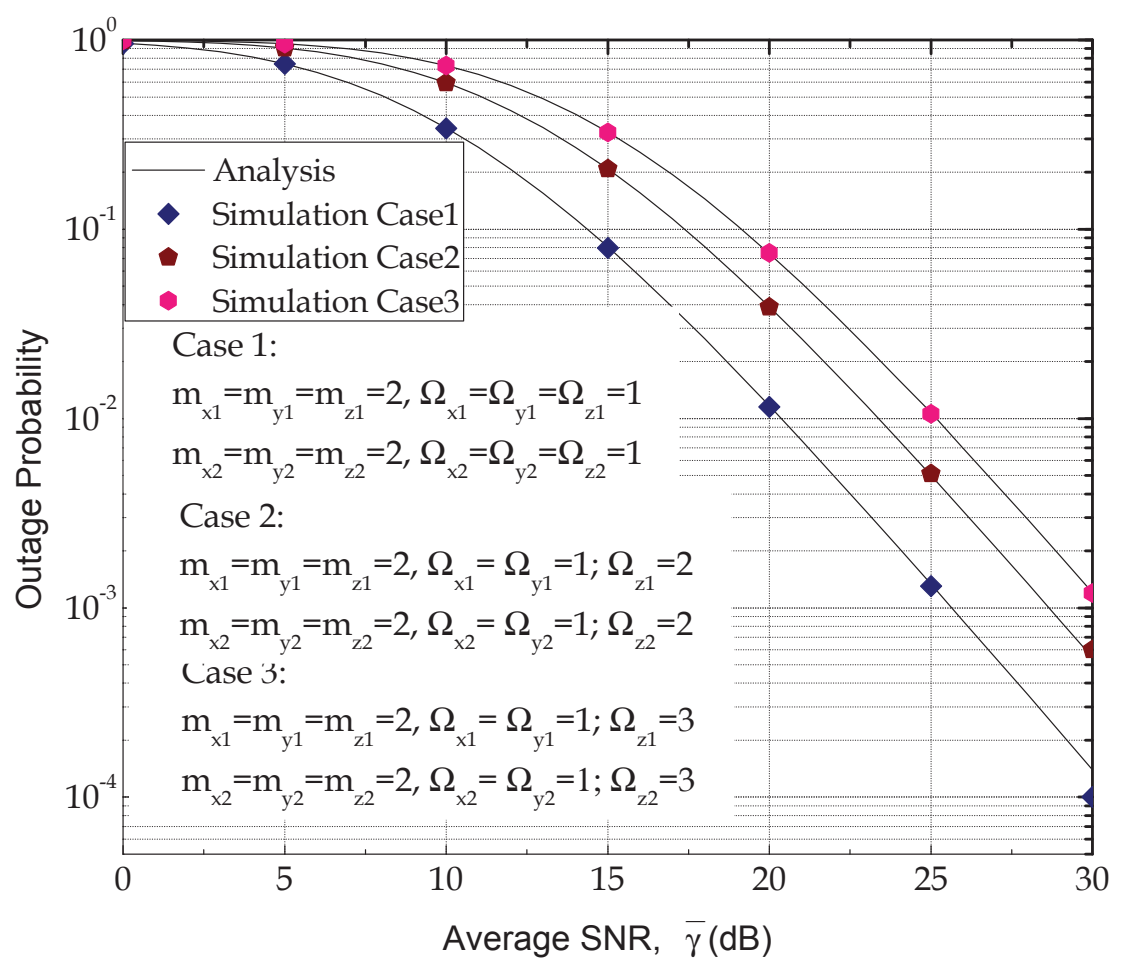

Figure 3.4: OP versus average SNR when Case1: $m_{X_{1}}=m_{X_{2}}=m_{Y_{1}}=m_{Y_{2}}=m_{Z_{1}}=m_{Z_{2}}=2$, $\Omega_{X_{1}}=\Omega_{Y_{1}}=\Omega_{Z_{1}}=\Omega_{X_{2}}=\Omega_{Y_{2}}=\Omega_{Z_{2}}=1$; Case2: $m_{X_{1}}=m_{X_{2}}=m_{Y_{1}}=m_{Y_{2}}=m_{Z_{1}}=m_{Z_{2}}=$ 2, $\Omega_{X_{1}}=\Omega_{X_{2}}=\Omega_{Y_{1}}=\Omega_{Y_{2}}=1, \Omega_{Z_{1}}=\Omega_{Z_{2}}=2$ and Case3: $m_{X_{1}}=$ $m_{X_{2}}=m_{Y_{1}}=m_{Y_{2}}=m_{Z_{1}}=m_{Z_{2}}=2, \Omega_{X_{1}}=\Omega_{X_{2}}=\Omega_{Y_{1}}=\Omega_{Y_{2}}=1, \Omega_{Z_{1}}=\Omega_{Z_{2}}=3$

nel power of the interference links $Y$ and $Z$, the PU-Tx is considered as the source of interference at the SU-Rx while the transmit power at the SU-Tx is degraded as the transmission is depending on power constraints.

In Fig. 3.4, the channel power of interference link $Z$ is increased to 2, in Case 2 and then to 3 , in Case 3. The result shows that Case 1 will still perform better than Case 2 and Case 3. The channel power of interference link $Y$ was decreased to 1 in all cases and the figure shows that decreasing the value of interference link $Y$, it has a positive impact on the signal as the outage probability of interference links $Y$ and $Z$ will also decrease. At $10^{-2}$ of outage probability, Case 1 has $20.1 \mathrm{~dB}$ of average SNR, Case 2 has $20.7 \mathrm{~dB}$ of average SNR while Case 3 is at $25 \mathrm{~dB}$ of average SNR. When the channel power of interference link $Z$ has been increased, the interference at the PU-Rx increases also. In this case the transmit powers at SU-Tx and SU-Relay are controlled by power constraints, which makes the transmission very difficulty.

By comparing these three figures above, we can say that, whenever the channel power is increased especially the channel powers of the interference links, the performance decreases more and more. Better performance is obtained whenever the channel 
powers of interference links are kept lower than when are higher. The analytical results match with the simulation results carried out with the help of Matlab. 


\section{Chapter 4}

\section{Conclusion}

In this thesis, spectrum sharing and its concepts have been considered and explained. In the last few years, research has shown that most radio frequency spectrum bands are not efficiently utilized and as a solution the cognitive radio network has been proposed in order to deliver better performance. The effect of primary network on spectrum sharing system over Nakagami- $m$ fading channel has been examined and an analytical expression for outage probability has been derived for Nakagami- $m$ fading channels. As the SU transmit power is controlled by the interference from the PU, this affects the performance of the cognitive radio network. The outage probability expression derived during this thesis can help us to evaluate the effects of primary network on the secondary network with AF relay to improve the performance of the network. The spectrum sharing system is proposed as a solution of how PU and SU can share the same frequency by keeping the interference from PU below a certain threshold. 



\title{
Abbreviations
}

\author{
AF Amplify and Forward \\ CDF Cumulative Density Function \\ CDMA Code Division Multiple Access \\ DF Decode and Forward
}

DSA Dynamic Spectrum Access

FCC Federal Communications Commission

MIMO Multiple-Input Multiple-Output

OFCOM Office of Communications

OFDM Orthogonal Frequency Division Multiplexing

PDF Probability Density Function

PU Primary User

SDR Software Defined Radio

SNR Signal to Noise Ratio

SU Secondary User

SU-Tx Secondary User-Transmitter

SU-Rx Secondary User-Receiver

TDMA Time Division Multiple Access

WCDMA Wideband Code Division Multiple Access

WiMAX Worldwide Interoperability for Microwave Access 



\section{Bibliography}

[1] F. K. Jondral: "Software-defined radio: Basics and evolution to cognitive radio," EURASIP Journal on Wireless Communications and Networking, vol. 5 no. 3, pp. 275-283, 1 August 2005.

[2] J. O. Neel, J.H. Reed, R.P. Gilles: "Convergence of cognitive radio networks," Wireless Communications and Networking Conference,Blacksburg, VA, U.S.A., vol. 4, pp. 2250-2255, March 2004.

[3] S. Haykin: "Cognitive radio: brain-empowered wireless communications," IEEE Journal, Ontario, Canada, vol. 23, no. 2, pp. 201-220, February 2005

[4] J. Mitola: "Cognitive radio: An integrated agent architecture for software defined radio," PHD Thesis, Royal Institute of Technology (KTH), Sweden, 2000.

[5] T.E. Hunter, A. Hedayat: "Cooperative communication in wireless networks," Communications magazine, IEEE, Dallas, U.S.A., vol. 42, no.10, pp. 74-80, October 2004.

[6] H. Kim, K. G. Shin: "In-band spectrum sensing in IEEE 802.22 WRANs for incumbent protection," IEEE trans. Mobile Computing, MI, U.S.A., vol.9, no 12, pp. 1766-1779, December 2010.

[7] R. Kaniezhil, C. Chandrasekar: "Performance analysis of wireless network with opportunistic spectrum sharing via cognitive radio nodes," Journal Elect. of Science and Technology, India, vol.10, no. 4, pp. 1766-1779, December 2012.

[8] N. Devroye, P. Mitran, V. Tarokh: "Achievable rates in cognitive radio channels," IEEE trans. Information theory, MA, U.S.A., vol. 52, no. 5, pp. 1813-1827, May 2006.

[9] J.M. Peha: "Approaches to spectrum sharing," IEEE Comm. Magazine, vol. 43, no. 2, pp. 10-12, February 2005.

[10] I. F. Akyildiz, W. Y. Lee, V. C. Mehmet, S. Mohanty: "A survey on spectrum management in cognitive radio networks," IEEE Comm. Magazine, Atlanta, U.S.A., Vol. 46, no. 4, pp. 40-48, April 2008. 
[11] A. Goldsmith, S. A. Jafar, I. Maric, S. Srinivasa: "Breaking spectrum gridlock with cognitive radios: An information theoretic perspective," Proc. IEEE, Vol. 97, no. 5, pp. 894-914, May 2009.

[12] A. Ghasemi, E.S. Sousa: "Fundamental limits of spectrum-sharing in fading environments," IEEE Trans. Wireless Communications, Ontario, Canada, vol. 6, no. 2, pp. 649-658, Feb 2007.

[13] M.G. Khoshkholgh, K. Navaie, H. Yanikomeroglu: "Access strategies for spectrum sharing in fading environment: Overlay, underlay and mixed," IEEE Trans. Mobile Computing, Tehran, Iran, vol. 9, no. 12, pp. 1780-1793, December 2010.

[14] Hung Tran: "Performance analysis of cognitive radio networks with interference constraints," Doctoral Dissertation Series No 2013:03, Blekinge Institute of Technology (BTH), Sweden, 2013.

[15] R. G.Vaughan, J. Andersen: "Antenna diversity in mobile communications," Electron. Lett., Lower Hutt, New Zealand, vol. 36, no. 4, pp. 149-172, November 1987.

[16] G. Andrieux, J. F. Diouris, Y. Wang: "A comparison between spatial and polarization diversity for transmit beamforming techniques," European Conference on Wireless Communication, Paris, France, pp. 161-164, October 2005.

[17] D. da Costa, H. Ding, J. Ge: "Interference-limited relaying transmissions in dualhop cooperatives networks over Nakagami-m fading," IEEE Commun. Lett., vol. 15, no. 5, pp. 1-3, 2011.

[18] J. Si, Li, Zan, X. Chen, B. Hao, Z. Liu: “On the performance of cognitive relay networks under primary user's outage constraint," IEEE Commun. Lett., vol. 15, no. 4, pp. 422-424, April 2011.

[19] L. Luo, P. Zhang, G. Zhang, J. Qin: "Outage performance of cognitive relay networks with underlay spectrum sharing," IEEE Commun. Lett., vol. 15, no. 7, pp. 710-712, July 2011.

[20] T. Q. Duong, V. N. Q. Bao, H.-J. Zapernick: "Exact outage probability of cognitive AF relaying with underlay spectrum sharing," Electron. Lett., vol. 47, no. 17, pp. 1001-1002, August 2011.

[21] T. Q. Duong, V. N. Q. Bao, H. Tran, G. C. Alexandropoulos, H.-J. Zapernick: "Effect of primary network on performance of spectrum sharing AF relaying," Electron. Lett., vol. 48, no. 1, pp. 25-27, January 2012.

[22] I. S. Gradshteyn, I. M. Ryzhik: "Table of integrals, series and products," Academic Press, 6th ed. San Diego, CA: Academic 2000. 